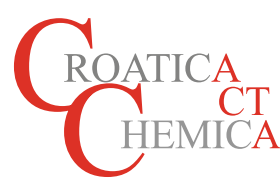

\title{
Crystal Structures of the Single PDZ Domains from GRASP65 and their Interaction with the Golgin GM130
}

\author{
Claudia M. Jurk, ${ }^{1}$ Yvette Roske, ${ }^{1}$ Udo Heinemann ${ }^{1,2, *}$
}

\author{
1 Macromolecular Structure and Interaction Laboratory, Max-Delbrück-Center for Molecular Medicine, Robert-Rössle-Straße 10, 13125 Berlin, Germany \\ 2 Institute of Chemistry and Biochemistry, Freie Universität Berlin, Takustraße 6, 14195 Berlin, Germany \\ * Corresponding author's e-mail address: heinemann@mdc-berlin.de \\ RECEIVED: April 12, 2018 * REVISED: June 19, 2018 * ACCEPTED: June 19, 2018
}

THIS PAPER IS DEDICATED TO DR. BISERKA KOJIĆ-PRODIĆ ON THE OCCASION OF HER 80 ${ }^{\mathrm{TH}}$ BIRTHDAY

\begin{abstract}
Among the major components of the Golgi apparatus are the GRASP family proteins, including GRASP65 on the cis-Golgi side. With its GRASP domain, GRASP65 is involved in Golgi stacking and ribbon formation. Interaction of GRASP65 with the Golgi marker protein GM130 is important for the docking of vesicles to the Golgi membrane. We present here structures of the two individual PDZ domains comprising the GRASP domain in human GRASP65. We use isothermal titration calorimetry to probe the interaction between GRASP65 and GM130. Additionally, we present evidence for the limited sequence conservation of the PDZ fold by describing the PDZ domain structure of the GRASP65 homolog Grh1 from Saccharomyces cerevisiae.
\end{abstract}

Keywords: PDZ domain structure, Golgi stacking, GRASP family, Golgins, Golgi apparatus, yeast homolog of GRASP65, vesicle transport, Grh1.

\section{INTRODUCTION}

A $S$ part of the endomembrane system of eukaryotic cells, the Golgi apparatus is traversed by proteins originating from the endoplasmic reticulum (ER) as they translocate to their final destination in or beyond the plasma membrane. It is composed of cisternal membrane structures arranged in Golgi stacks which are further organized into a larger ribbon structure. ${ }^{[1]}$ The Golgi apparatus has three primary compartments, known as "cis" (cisternae nearest the endoplasmic reticulum), "medial" (central layers of cisternae), and "trans" (cisternae farthest from the endoplasmic reticulum). The cis-Golgi network is the entry area at the convex side of the Golgi stack which faces to the ER whereas the trans-Golgi network is the concave side that directs newly synthesized proteins to different subcellular destinations.

Proteins of the GRASP (Golgi reassembly stacking protein) and golgin families are part of the structural organization of the Golgi apparatus. ${ }^{[2,3]}$ Two proteins involved in mammalian Golgi stacking and laterally linking the Golgi cisternae to form Golgi ribbons are GRASP65 and GRASP55. ${ }^{[4,5]}$ GRASP55, anchored to the medial Golgi membrane by myristoyl and palmitoyl groups, interacts with the golgin protein Golgin-45.[6] GRASP65, differing in localization, is $\mathrm{N}$-terminally associated with the membranes of the cis Golgi through a myristoyl group. Interaction with the C-terminus of the Golgi marker protein GM130 recruits GRASP65 to the cis-Golgi membrane where it is involved in the docking of transport vesicles to the Golgi membrane. ${ }^{[5,7,8]}$

The human GRASP65 (hGRASP65) protein contains two PDZ domains (PDZ1, PDZ2) at the N-terminus, which, together, represent the entire GRASP domain (Figure 1A). The PDZ domains of GRASP65, especially the PDZ1 domain, are known to be essential for self-association of the protein and for initiating the linking of Golgi cisternae into mini stacks. ${ }^{[9-11]}$ Initial biochemical studies suggested that the binding motif for GM130 is found within the second PDZ domain of GRASP65, but recent crystallographic studies of

(c) B. BY This work is licensed under a Creative Commons Attribution 4.0 International License. 
A

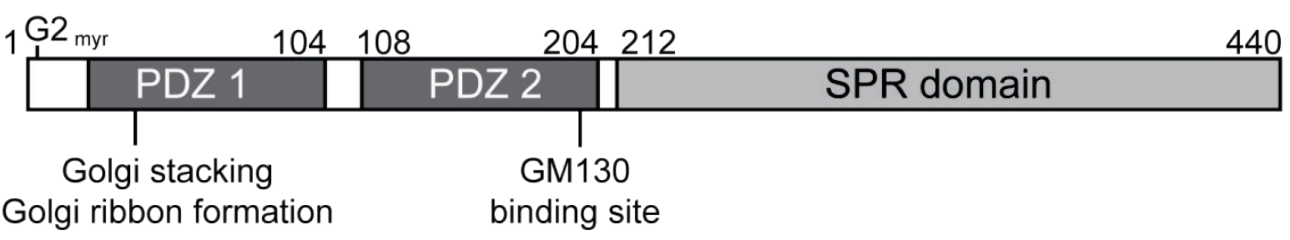

B

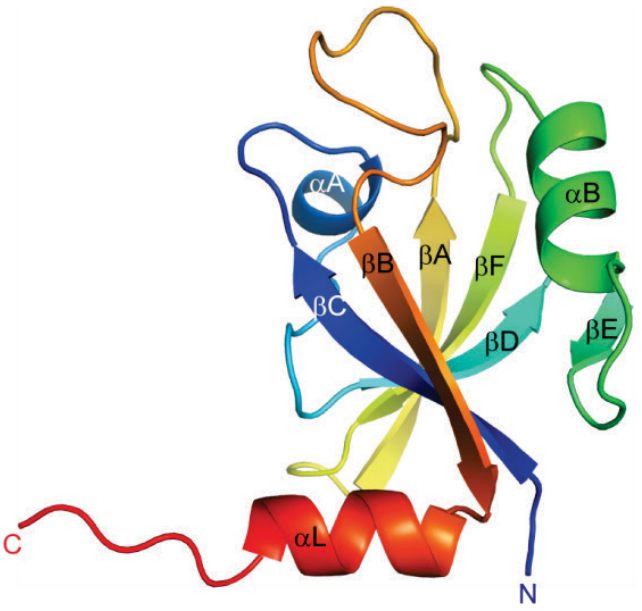

$\mathrm{D}$

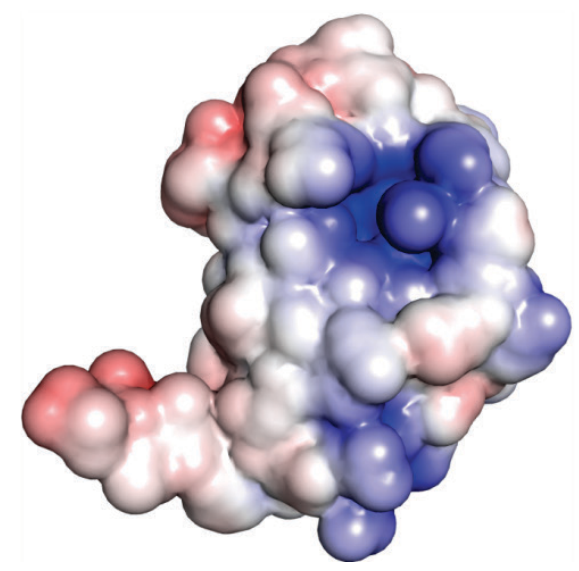

C
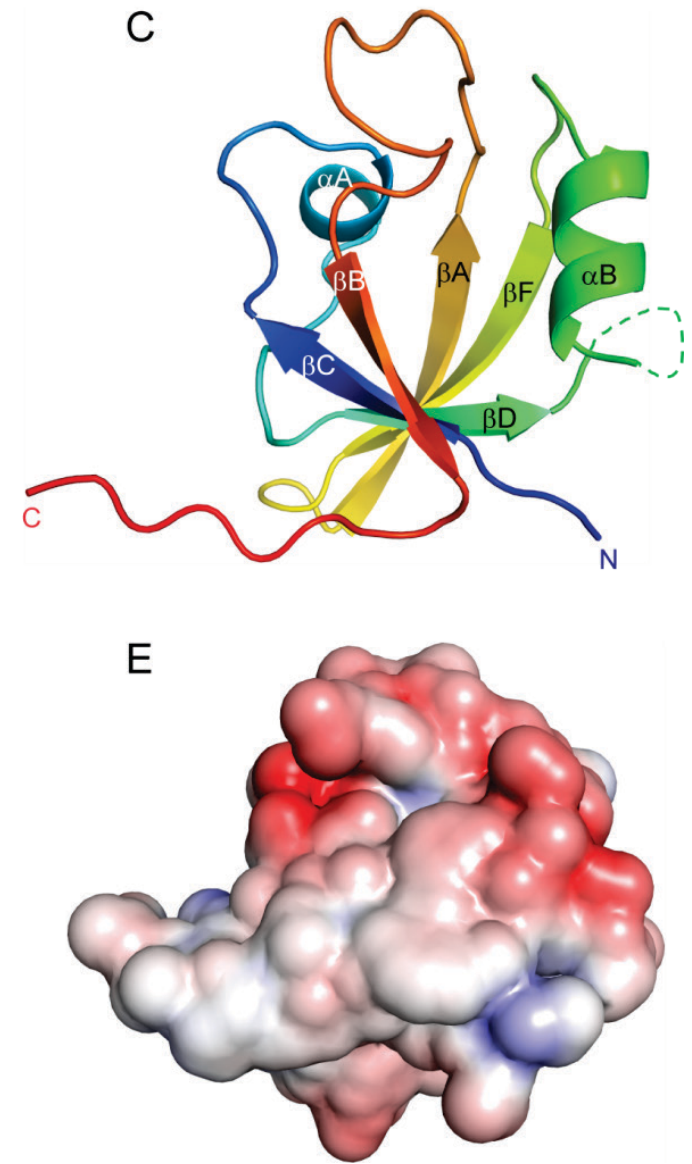

Figure 1. Domain architecture and PDZ structures of GRASP65. (A) Domain organization and boundaries of GRASP65. The two $\mathrm{N}$-terminal PDZ domains are shown in dark gray and the C-terminal serine/proline-rich domain (SPR) in light gray. The two PDZ domains, residues 1-204, comprise the GRASP domain, which is anchored to the Golgi membrane via its myristoylated Nterminal glycine (G2 myr). The crystal structure of monomeric GRASP65 PDZ1 is shown in (B) and of GRASP65 PDZ2 in (C). The cartoon representations are color-coded from the $\mathrm{N}$-terminus in blue to the $\mathrm{C}$-terminus in red. Non-resolved amino acids are indicated by a dashed line. The electrostatic surface representations for GRASP65PDZ1 (D) and GRASP65PDZ2 (E) are shown in the same orientation as the structures above and were calculated by the Adaptive Poisson-Boltzmann Solver implemented in the Pymol software. ${ }^{[41]}$ Blue indicates positive charge and red negative charge at the level of $\pm 5 \mathrm{kT} / \mathrm{e}$.

the hGRASP65 GRASP domain bound to the C-terminal tail of GM130 showed that both PDZ domains are involved in GM130 binding. ${ }^{[12]}$ The C-terminal region of GRASP65 consists of an SPR (serine/proline rich) domain which is a target for multiple phosphorylation during mitosis. ${ }^{[13]}$

PDZ domains comprise 80-100 residues and are often found in multi-domain proteins used as scaffolds, especially in signal transduction complexes. PDZ domains were initially characterized in the post-synaptic density protein PSD95, the Drosophila disc large tumor suppressor (Dlg1) and zona occludens-1 protein (ZO-1), and their name is derived from these three proteins. Almost all PDZ domains share a $\beta$-sandwich fold containing six $\beta$-strands framed by two $\alpha$-helices. ${ }^{[14,15]}$ A common feature of PDZ 
domains is their ability to bind the C-terminus of protein ligands in a hydrophobic binding pocket in addition to the binding by a highly conserved carboxylate-binding loop, which is also involved in the binding of non-peptide inhibitors. ${ }^{[16]}$ Binding of internal sequences in the peptidebinding pocket has also been reported. ${ }^{[15,17]}$

To understand the binding of GM 130 by GRASP 65 in more detail, we studied GRASP65 binding of GM130 derived peptides by isothermal titration calorimetry (ITC) in conjunction with structural analysis. For these studies, we used different GRASP65 constructs, comprising the individual PDZ domains, the entire GRASP domain or an Nterminally truncated GRASP domain. We present here the crystal structures of the single PDZ domains of GRASP65 as well the first PDZ domain structure of the GRASP65 yeast homolog, Grh1.

\section{MATERIALS AND METHODS}

\section{Protein Expression and Purification}

Fragments of the human grasp65 gene (gene ID: 64689) encoding amino acid residues (aa) 1-118 (GRASP65 ${ }^{\text {PDZ1) }}$ ), aa 108-204 (GRASP65 ${ }^{\text {PDZ2 }) ~ a n d ~ a a ~ 1-204 ~(G R A S P 65-204), ~ a n d ~ o f ~}$ the Saccharomyces cerevisiae gene grh1 (gene ID: 852129) encoding the fragment aa 67-144 (GRH1 $\left.{ }^{\mathrm{PDZ} 1 \Delta \beta}\right)$ were inserted into a pET-46 Ek/LIC vector using the ligation independent cloning (LIC) technique ${ }^{[18]}$ according to the manufacturer's instructions (Novagen). Additionally, a PreScission protease recognition sequence (LEVLFQGP) was introduced at the $5^{\prime}$-end of the construct. Template cDNA of human grasp65 was kindly provided by Erich Wanker (MDC Berlin-Buch, Germany). Yeast cDNA, used as a template, was kindly provided by Thomas Sommer (MDC Berlin-Buch, Germany).

For recombinant protein expression, E. coli T7 Express Rosetta 2 cells (New England Biolabs) were transformed with the $\mathrm{pET}-46 \mathrm{Ek} / \mathrm{LIC}$ plasmid harboring the grasp65 or grh1 constructs. All constructs were expressed as fusion proteins with an $\mathrm{N}$-terminal $\mathrm{His}_{6}$ tag. Cells were grown in Superior Broth (SB) medium supplemented with the appropriate antibiotics at $37{ }^{\circ} \mathrm{C}$ to an $\mathrm{OD}_{600}$ (optical density at $600 \mathrm{~nm}$ ) of 1 . Cells were then cooled down to 20 ${ }^{\circ} \mathrm{C}$, and gene expression was induced with $1 \mathrm{mM}$ isopropyl$\beta$-D-thiogalactopyranoside (IPTG). $16 \mathrm{~h}$ after induction, cells were harvested, resuspended in lysis buffer $(20 \mathrm{mM}$ HEPES, pH 8.0, $500 \mathrm{mM} \mathrm{NaCl}, 10 \%$ glycerol, $10 \mathrm{mM}$ imidazole) and lysed with a Sonoplus sonifier (BANDELIN electronic).

Proteins were purified by affinity chromatography with an Äkta (GE Healthcare) purification system using a HisTrap ${ }^{\text {TM }}$ FF (GE Healthcare) column. After the wash step (20 mM HEPES, pH 8.0, $500 \mathrm{mM} \mathrm{NaCl}, 40 \mathrm{mM}$ imidazole), protein was eluted with $250 \mathrm{mM}$ imidazole and incubated with PreScission protease to cleave off the $\mathrm{His}_{6}$ tag overnight while dialyzing against a 100 fold excess of 20 mM HEPES, pH 8.0, $250 \mathrm{mM} \mathrm{NaCl}$ and $2 \mathrm{mM} \beta$-mercaptoethanol. For tag and protease removal, a second affinity chromatography step was used. Finally, size exclusion chromatography on a HiLoad 26/60 Superdex 75 gel filtration column (GE Healthcare) was performed in a buffer containing $20 \mathrm{mM}$ HEPES, pH 8.0, $250 \mathrm{mM} \mathrm{NaCl}, 1 \mathrm{mM}$ EDTA and $2 \mathrm{mM}$ 2-mercaptoethanol. Peak fractions were concentrated to $10-15 \mathrm{mg} / \mathrm{ml}$. In contrast to the GRASP65 constructs, the tag was not removed for Grh1 ${ }^{\text {PDZ1 } 1 \triangle \beta}$ due to subsequent protein instability. Site-directed mutagenesis was performed using the QuikChange ${ }^{\circledR}$ Site-Directed Mutagenesis Kit (Stratagene, USA) according to the manufacturer's instructions.

\section{Crystallization}

Crystals were grown by vapor diffusion using the sitting drop method with a semi-automated dispensing system (Hydra II, Matrix). GRASP65PDZ1 crystals were obtained within $24 \mathrm{~h}$ in $24 \%$ PEG1500 and $20 \%$ glycerol at $20^{\circ} \mathrm{C}$. GRASP65 ${ }^{\text {PDZ2 }}$ crystals appeared in 2-3 weeks in a condition containing $1.4 \mathrm{M} \mathrm{Na} / \mathrm{K}$ phosphate, $\mathrm{pH} 8.2$, also at $20{ }^{\circ} \mathrm{C}$. Grh1 $1^{\mathrm{PDZ} 1 \triangle \beta}$ crystals were obtained within 3-4 weeks in $3.0 \mathrm{M}$ $\mathrm{NaCl}$ and $100 \mathrm{mM}$ Bis-Tris, $\mathrm{pH} 5.5$ at $20^{\circ} \mathrm{C}$. The best crystals of GRASP65 ${ }^{\text {PDZ2 }}$ and Grh1 $1^{\text {PDZ1 } \beta}$ were flash frozen in mother liquor containing $20 \%$ PEG400 or $20 \%$ glycerol, respectively.

\section{Data Collection and Structure Determination}

Diffraction data were collected at a temperature of $100 \mathrm{~K}$ at beamline 14.1 at BESSY II (Helmholtz-Zentrum Berlin, HZB) in Germany. ${ }^{[19]}$ GRASP65 ${ }^{\text {PDZ1 }}$ data from a crystal in space group $C 222_{1}$ containing one protein molecule per asymmetric unit (AU) were collected at $100 \mathrm{~K}$ to a resolution of $2.7 \AA$, and GRASP $65^{\text {PDZ2 }}$ data were obtained up to $2.1 \AA$ resolution from a space group $C 2$ crystal with two protein molecules per asymmetric unit. Data were processed using XDS. ${ }^{[20,21]}$ Phases were obtained by molecular replacement with Phaser[22] from the CCP4 suite ${ }^{[23,24]}$ using the coordinates of the matching GRASP55 PDZ domain (PDB entry code: 3RLE) as search model. Subsequently, an atomic model was built and refined using COOT and REFMAC5. ${ }^{[25-28]}$

A 1.4 A native dataset was collected at a wavelength of $0.91841 \AA$ from a Grh1 ${ }^{\text {PDZ1 } \triangle \beta}$ crystal at beamline MX14.2 at BESSY. Additionally, a $1.75 \AA$ data set collected at $1.77122 \AA$ from a crystal obtained under the same crystallization condition was used for an S-SAD experiment. Both data sets were processed using XDS, with the keyword 'FRIEDEL'S_LAW=FALSE' for the $1.75 \AA$ data set. ${ }^{[20,21]}$ Both crystals belonged to space group $P 3_{1} 21$. Based on the 
Table 1. Data collection and refinement statistics

\begin{tabular}{|c|c|c|c|c|}
\hline & GRASP65 & GRASP65 & Native Grh1 $1^{\text {PDZ1 } 1 \beta}$ & S-SAD Grh1 $1^{\text {PDZ1 } \triangle \beta}$ \\
\hline \multicolumn{5}{|l|}{ Data collection } \\
\hline Wavelength & 0.91841 & 0.91841 & 0.91841 & 1.77122 \\
\hline Space group & $C 222_{1}$ & $C 2$ & $P 3_{1} 21$ & $P 3_{1} 21$ \\
\hline \multicolumn{5}{|l|}{ Cell dimensions } \\
\hline$a, b, c(\AA)$ & $46.4,63.2,80.8$ & $50.8,82.6,49.2$ & $53.4,53.4,50.9$ & $53.5,53.5,51.0$ \\
\hline$\alpha, B, \gamma\left(^{\circ}\right)$ & $90.0,90.0,90.0$ & $90.0,110.8,90.0$ & $90.0,90.0,120.0$ & $90.0,90.0,120.0$ \\
\hline \multirow[t]{2}{*}{ Resolution $(\AA ̊)^{*}$} & $33.93-2.67$ & $26.85-2.12$ & $26.72-1.40$ & $34.28-1.75$ \\
\hline & $(2.74-2.67)$ & $(2.18-2.12)$ & $(1.50-1.40)$ & $(1.84-1.75)$ \\
\hline$R_{\text {meas }}^{(a)}(\%)^{*}$ & $6.2(75.5)$ & $7.8(62.8)$ & $4.2(61.5)$ & $5.3(70.5)$ \\
\hline Average $I / \sigma(l)^{*}$ & $20.7(2.3)$ & $15.8(3.0)$ & $22.96(3.4)$ & $36.55(3.2)$ \\
\hline Completeness (\%)* & $99.8(100.0)$ & $99.3(100.0)$ & $99.7(99.6)$ & $98.9(93.6)$ \\
\hline Multiplicity & 4.3 & 4.1 & 6.5 & 15.2 \\
\hline \multicolumn{5}{|l|}{ Refinement } \\
\hline No. reflections & 3394 & 10222 & 16072 & \\
\hline$R_{\text {work }}(\mathrm{b}) / R_{\text {free }}{ }^{(c)}(\%)$ & $20.9 / 25.8$ & $18.6 / 23.5$ & $16.3 / 21.0$ & \\
\hline \multicolumn{5}{|l|}{ No. atoms } \\
\hline Protein & 848 & 1378 & 801 & \\
\hline Ligands & 1 & 5 & 3 & \\
\hline Water & 9 & 96 & 141 & \\
\hline \multicolumn{5}{|l|}{ B-factors } \\
\hline Overall mean $\left(\AA^{2}\right)$ & 62.1 & 32.9 & 25.0 & \\
\hline \multicolumn{5}{|l|}{ R.m.s. deviation } \\
\hline Bond lengths (Å) & 0.007 & 0.020 & 0.010 & \\
\hline Bond angles $(\stackrel{\circ}{)})$ & 1.25 & 1.97 & 1.45 & \\
\hline \multicolumn{5}{|l|}{ Ramachandran map } \\
\hline Most favored (\%) & 97.12 & 94.74 & 100.00 & \\
\hline Additionally allowed & 2.88 & 5.26 & 0.00 & \\
\hline Disallowed (\%) & 0.00 & 0.00 & 0.00 & \\
\hline
\end{tabular}

anomalous scattering from three sulfur atoms present in two methionine residues and one cysteine residue, we could solve the phase problem by using the S-SAD method $^{[29,30]}$ of Auto-Rickshaw, an automated crystal structure determination platform. ${ }^{[31]}$ The polypeptide backbone could be traced automatically by ARP/WARP. ${ }^{[32]}$ Subsequently, an atomic model was built and refined against the native dataset using $\operatorname{COOT}^{[25,26]}$ and REFMAC5. ${ }^{[27,28]}$

All data collection and refinement statistics are given in Table 1. The final structures were validated using MolProbity. ${ }^{[33]}$ The atomic coordinates and structure factors for GRASP65 PDZ1 (code 6G8T), GRASP65 ${ }^{\text {PDZ2 (code }}$ 6G8W) and Grh1 ${ }^{\mathrm{PDZ1} 1} \beta$ (code $6 \mathrm{G} 8 \mathrm{Y}$ ) have been deposited in the Protein Data Bank (http://wwpdb.org/).

\section{Isothermal Titration Calorimetry (ITC)}

For ITC measurements, different GRASP65 constructs comprising the entire GRASP domain (aa 1-204) and the single PDZ domains were prepared as described above. Peptides of the C-terminus of GM130 were purchased in lengths of six and twelve amino acids from Peptide Specialty Laboratories (PSL, Heidelberg). To avoid any buffer mismatch between protein and peptide, all constructs and peptides were extensively dialyzed $(20 \mathrm{mM}$ HEPES, pH 8.0, $250 \mathrm{mM} \mathrm{NaCl}$ ). Prior to the ITC measurements the samples were degassed under vacuum with stirring using a ThermoVac (Microcal). The ITC measurements were run in a VP-ITC micro calorimeter (Microcal). The cell was loaded with $2 \mathrm{ml}$ of $75 \mu \mathrm{M}$ protein 
solution, and $1.5 \mathrm{mM}$ peptide solution was placed in the syringe. About 60 injections were performed with a volume of $2.5 \mu \mathrm{l}$ for the first and $5 \mu \mathrm{l}$ for the remaining injections. Time between injections was $240 \mathrm{~s}$. All experiments were conducted at $15{ }^{\circ} \mathrm{C}$ with a stirring speed of $307 \mathrm{rpm}$. Raw data were baseline fitted with NITPIC (version 1.2.2) ${ }^{[34]}$ and integrated using ORIGIN (version 7.0, OriginLab).

\section{RESULTS}

\section{Structures of the Single PDZ Domains of GRASP65}

The first PDZ domain of the GRASP domain comprises amino acid residuess 1-104 (Figure 1A). The $\mathrm{N}$-terminal amino acids 1-12 in the crystallized GRASP65 ${ }^{\text {PDZ1 }}$ construct were not explained by electron density and could not been modeled. The globular structure, as shown in Figure $1 \mathrm{~B}$, consists of six $\beta$-strands ( $\beta A-\beta F$ ) and three $\alpha$-helices ( $\alpha A, \alpha B$ and $\alpha \mathrm{L})$ arranged in a partially opened $\beta$-sandwich typical of PDZ domains. Helix $\alpha \mathrm{L}$ represents the linker connecting the first with the second PDZ domain of GRASP65. The structure exhibits, as other PDZ domains do, a ligandbinding pocket formed by strand $\beta B$ and helix $\alpha B$, exposing positively charged residues (Figure 1D).

The second PDZ domain, GRASP65 ${ }^{\mathrm{PDZ} 2}$, was also crystallized (Figure $1 C$ ) and also exhibited the typical PDZ fold consisting of five $\beta$-strands ( $\beta A-\beta D$ and $\beta F$ ) and two $\alpha$-helices $(\alpha \mathrm{A}$ and $\alpha \mathrm{B})$. In contrast to PDZ1, here the ligand-binding pocket shows a more hydrophobic character (Figure 1E).

As shown in the superposition of GRASP65 $5^{\text {PDZ1 }}$ and GRASP65 ${ }^{\text {PDZ2 }}$ (Figure 2A) both domains reveal a similar structure with an elongated carboxylate-binding loop between strands $\beta A$ and $\beta B$. The $C \alpha$ atom r.m.s. deviation is $0.78 \AA$ (calculated with PyMOL, DeLano Scientific, USA) despite a low sequence match of $35 \%$, shown by the sequence alignment (Figure 2B).

\section{Dimerization of the Single GRASP65 PDZ Domains}

Crystal structures of both monomeric PDZ domains of GRASP65 are illustrated in Figure 1. Size-exclusion chromatography, however, reveals formation of mainly tetramers for GRASP65 $5^{\mathrm{PDZ} 1}$ and dimers for GRASP65 $5^{\mathrm{PDZ2}}$ in solution (Figure $3 \mathrm{~A}, \mathrm{~B}$ ). Insight into the geometry of the arrangements may be obtained from the crystal packing. In both crystal structures, two protein molecules are forming a dimer via the peptide binding pocket. In GRASP65 ${ }^{\text {PDZ1, }}$ which crystallized in space group $C 222_{1}$ with one protein molecule per $\mathrm{AU}$, the dimer is formed via the twofold axis along the crystallographic $b$ axis. A tetrameric arrangement as observed in solution could not be found in the GRASP65 ${ }^{\mathrm{PDZ1}}$ crystal. The two molecules per AU, present in the space group $C 2$ crystals of GRASP65 ${ }^{\text {PDZ2 }}$, form a dimer

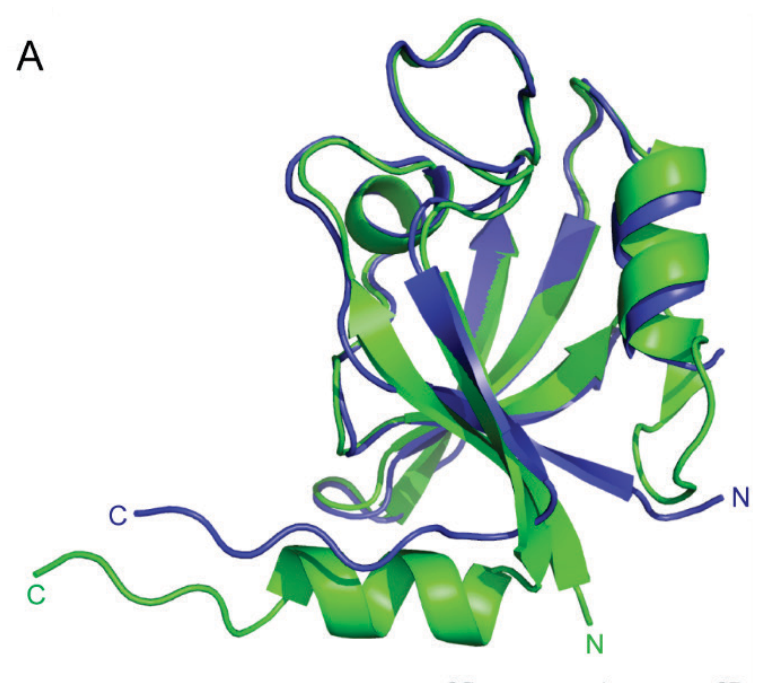

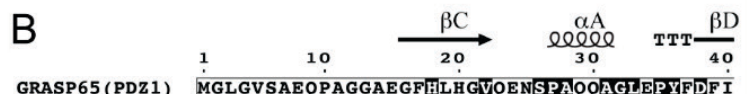
GRASP65(PDZ1)
GRASP65(PDZ2) MLGVSAEQPAGGAEGFHLHGVQENSPAQQAGLEPYFDFI

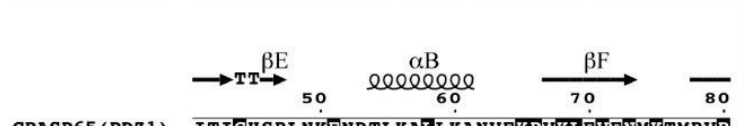
$\begin{array}{lll}\text { GRASP65 (PDZ1) } & \text { ITIGHSRLNKENDTLKALLKANVEKPVKLEVFNMKTMRVR } \\ \text { GRASP65(PDZ2) } & \text { V..GSDQILQESEDFTLIESHEGKRLKLMVYSSKSDSCR }\end{array}$

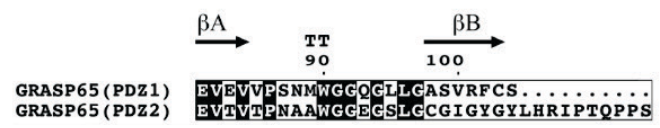

Figure 2. Superposition of GRASP65 ${ }^{\text {PDZ1 }}$ and GRASP65 ${ }^{\text {PDZ2 }}$. (A) Structure of GRASP65 ${ }^{\text {PDZ1 }}$ (green) is superimposed onto GRASP65 PDZ2 (blue) with an r.m.s.d. of $0.78 \AA$. (B) Sequence alignment of PDZ1 and PDZ2 of GRASP65 (with ESPript 2.2). ${ }^{[42]}$

via non-crystallographic symmetry (NCS). In both structures the C-terminus of molecule 1 form hydrogen bonds with the carboxylate binding loop of molecule 2, thus mimicking the classical PDZ-ligand interaction. For GRASP65 $5^{\mathrm{PDZ} 1}$ the Cterminal carboxylate group of Val118 of molecule 2 (Figure 3C) forms four hydrogen bonds with the backbone of residues Leu95, Leu96, Gly97 and Ala98 in the carboxylate binding loop of molecule 1 . An additional hydrogen bond is formed with the carbonyl oxygen of Ala98 and the backbone nitrogen of Val118 (Figure 3C).

In GRASP65 ${ }^{\text {PDZ2 }}$ the carboxylate group of the Cterminal residue Thr204 of molecule 2 also forms four hydrogen bonds with the backbone of residues Ser189, Leu190, Gly191 and Cys192 of molecule 1 with an extra hydrogen bond between the carbonyl oxygen of Cys192 and the backbone nitrogen of Thr204 (Figure 3D).

With $1027 \AA^{2}$, the dimer interface of GRASP65 $5^{\text {PDZ1 }}$ covers $9.5 \%$ of the total solvent-accessible surface (SAS) of both subunits $\left(\begin{array}{lll}10850 & \AA^{2}\end{array}\right)$. The dimer interface of 
A

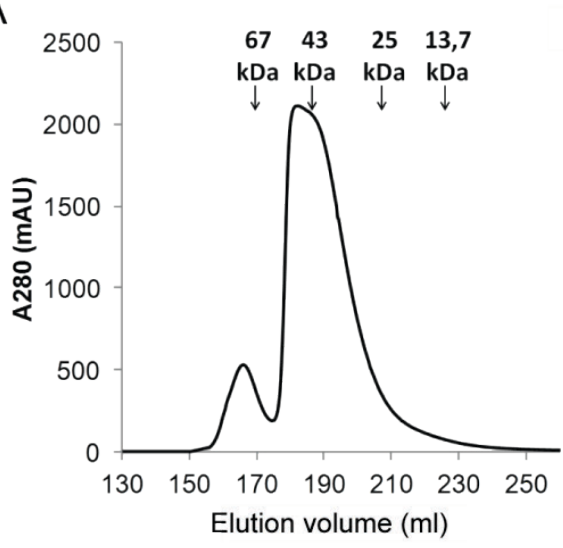

B

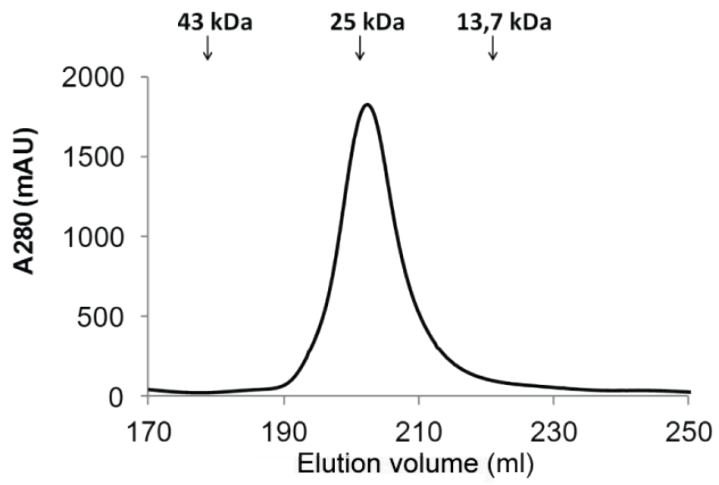

C
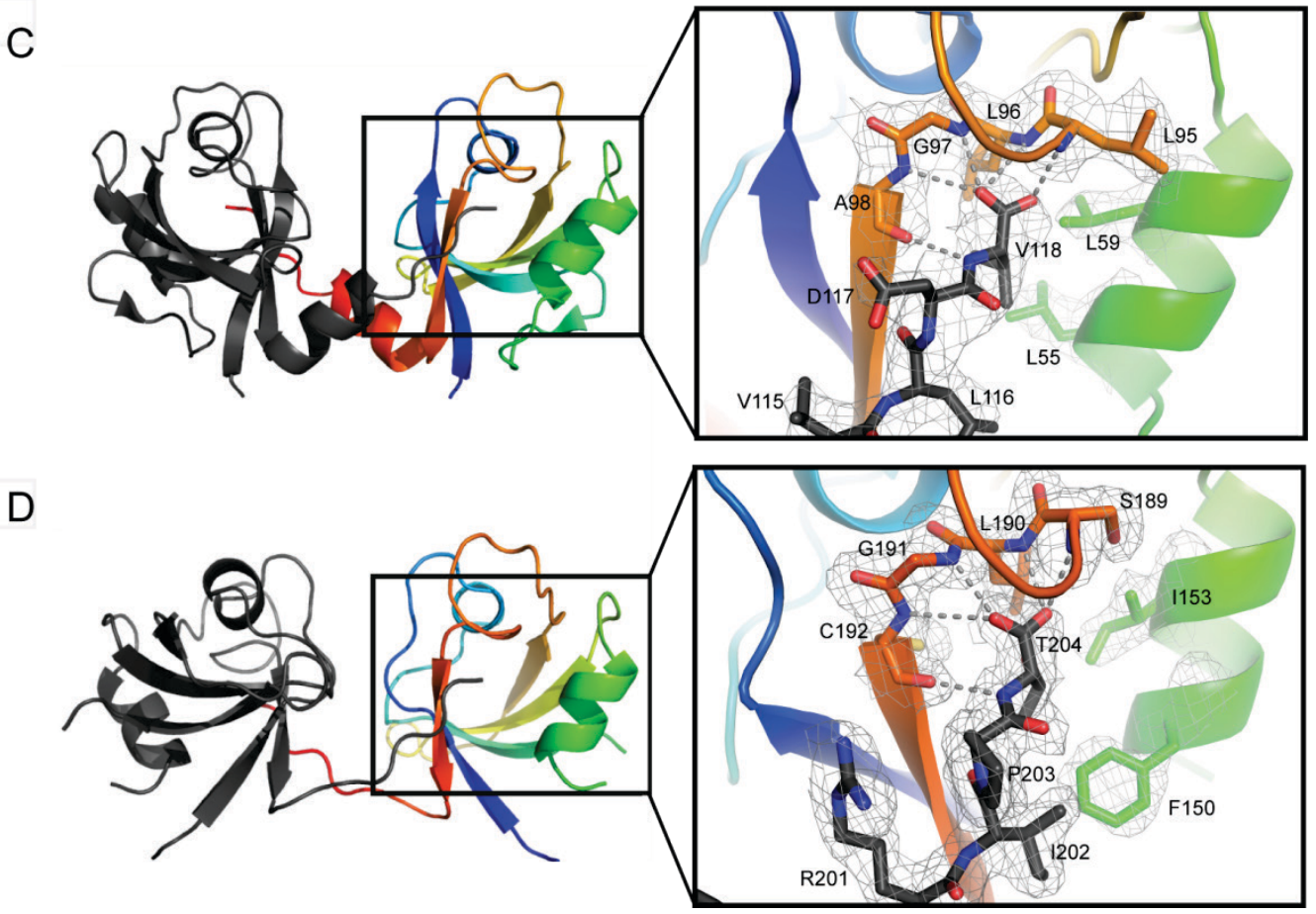

Figure 3. Oligomerization of the single GRASP65 PDZ domains. (A) Elution profile of GRASP65PDZ1 shows a main peak in size exclusion chromatography at $\sim 50 \mathrm{kDa}$, which corresponds to a tetramer size of GRASP65 ${ }^{\text {PDZ1 }}$ (molecular mass of monomer is $13 \mathrm{kDa}$ ). A small portion of higher oligomers is visible at $\sim 80-90 \mathrm{kDa}$. (B) The elution profile of GRASP65PDZ2 shows a peak at $\sim 20$ $\mathrm{kDa}$, which indicates dimer formation. The size of the molecular weight standards is given above the elution profiles. Protein

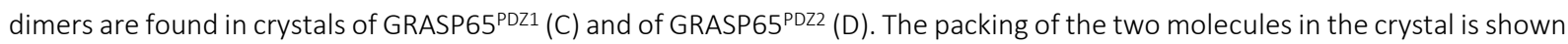
on the left. Molecule 1 is color-coded from the $\mathrm{N}$-terminus (blue) to the C-terminus (red). Molecule 2 is depicted in gray. The ligand-binding pocket is shown in detail in the magnified inset. Residues contributing in the dimer formation are shown as stick model with surrounding $2 F_{0}-F_{c}$ electron density contoured at 1 sigma. Hydrogen bonds are represented as dashed lines.

GRASP65 $5^{\text {PDZ2 }}$ covers $8.5 \%$ of the SAS $\left(832 \AA^{2}\right.$ of $9800 \AA^{2}$ in total) as calculated using PDBe PISA. ${ }^{[35]}$

\section{Interaction of the GRASP Domain with GM130}

In order to analyze the binding of GRASP65 to the golgin GM130 in greater detail, different GRASP65 constructs were prepared comprising either the entire GRASP domain (GRASP65 $5^{1-204}$ ) or the single PDZ domains (GRASP65 ${ }^{\text {PDZ1 }}$ and GRASP65 ${ }^{\text {PDZ2 }}$ ). The single PDZ domains and GRASP65 ${ }^{1-204}$ were titrated in the ITC experiment with the hexapeptide NVKITVIC representing the $C$ terminus of GM130. A dodecameric $\mathrm{GM} 130$ peptide (NADENDEVKITVIC) was only titrated to GRASP651-204. GRASP65 ${ }^{\text {PDZ2 }}$ alone showed no binding of 
VKITVI (Figure 4B), whereas GRASP65 ${ }^{\text {PDZ1 }}$ did, indeed, show binding of the hexapeptide (Figure 4A). The ITC data could only be fitted by using a 4-site sequential binding model, indicating that four peptides associate with GRASP65 $5^{\text {PDZ1 }}$. Due to our observation that the isolated PDZ1 domain of GRASP65 elutes as tetramer in size exclusion chromatography, we assume that the binding of the peptide may cause a dissociation of the tetramer, possibly triggered by conformational changes in the subunits of the multimeric protein. Therefore we could only fit the data to a sequential binding model with a resulting overall dissociation constant of $K_{D}=41.8 \pm 3.0 \mu \mathrm{M}$. It is not possible to obtain a dissociation constant for each binding site for this sequential binding site model, because all possible states of association exist in equilibrium. The titration of the VKITVI hexapeptide to the construct GRASP65 $5^{1-204}$ revealed a $K_{D}$ of $25.1 \pm 0.6 \mu \mathrm{M}$ (Figure $4 \mathrm{C}$ ). The GM130 dodecapeptide ADENDEVKITVI showed binding of the entire GRASP domain (GRASP65 ${ }^{1-204}$ ) with a $K_{D}$ of $27.6 \pm$ $0.6 \mu \mathrm{M}$ (Figure 4D). In both cases, the binding stoichiometries ( $N$ values) differ from 1:1 and are difficult to explain. In the absence of other evidence, we cannot exclude the possibility that the complex stoichiometries reflect incomplete binding of the ligand peptides to protein domains whose binding sites are partially occupied by terminal peptides from other protein chains, in a similar way as seen in the crystals.

\section{Crystal Structure of Grh1, the Yeast Homolog of GRASP65}

In addition to the GRASP65 PDZ domain structures, the structure of the first PDZ domain, Grh1 $1^{\text {PDZ1 } \triangle \beta B}$ (aa 67-144),

of the GRASP65 yeast homolog Grh1 $1^{[36,37]}$ was determined using the sulfur SAD method. ${ }^{[25,26]}$ The first PDZ domain of Grh1 consists of four $\beta$-strands ( $\beta A, \beta C, \beta D$ and $\beta F$ ), three $\alpha$-helices $(\alpha A, \alpha B$ and $\alpha P)$ and an extended loop region connecting strand $\beta D$ with helix $\alpha B$ (Figure $5 A$ ). Although the crystallized fragment lacks the carboxylate-binding loop and the central strand $\beta B$, the structure exhibits a PDZ-like fold. These two C-terminal structural elements were omitted from the cloned construct due to issues of low solubility or low level of overexpression encountered with longer protein constructs. This becomes obvious in the superposition of GRASP65 $5^{\mathrm{PDZ1}}$ and Grh1 $1^{\mathrm{PDZ} 1 \triangle \beta \mathrm{B}}$ (Figure 5B). In spite of the very low sequence identity of $10 \%$, the PDZ structures superimpose quite well (r.m.s.d. $=1.4 \AA$ for $\mathrm{C} \alpha$ atoms) (Figure $5 \mathrm{C}$ ). That proves that similar PDZ folds can be adopted by sequentially distant protein domains.

\section{DISCUSSION}

We present the crystal structures of the two separate PDZ domains of human GRASP65, GRASP65 PDZ1 and GRASP65 ${ }^{\text {PDZ2 }}$. Notably, both structures reveal a variation of the canonical PDZ fold, which is reflected in the sequential order of the secondary structure elements. As already shown for GRASP55, ${ }^{[38]}$ also GRASP65 contains two circularly permuted PDZ domains revealing the secondary structure organization $\beta \alpha \beta \alpha \beta \beta \beta$ typical of bacterial PDZlike domains instead of the $\beta \beta \beta \alpha \beta \beta \alpha \beta$ pattern normally found in mammals, such as human PSD-95. ${ }^{[14]}$ We observed this $\beta \alpha \beta \alpha \beta \beta \beta$ permutation also in Grh1, the yeast homolog
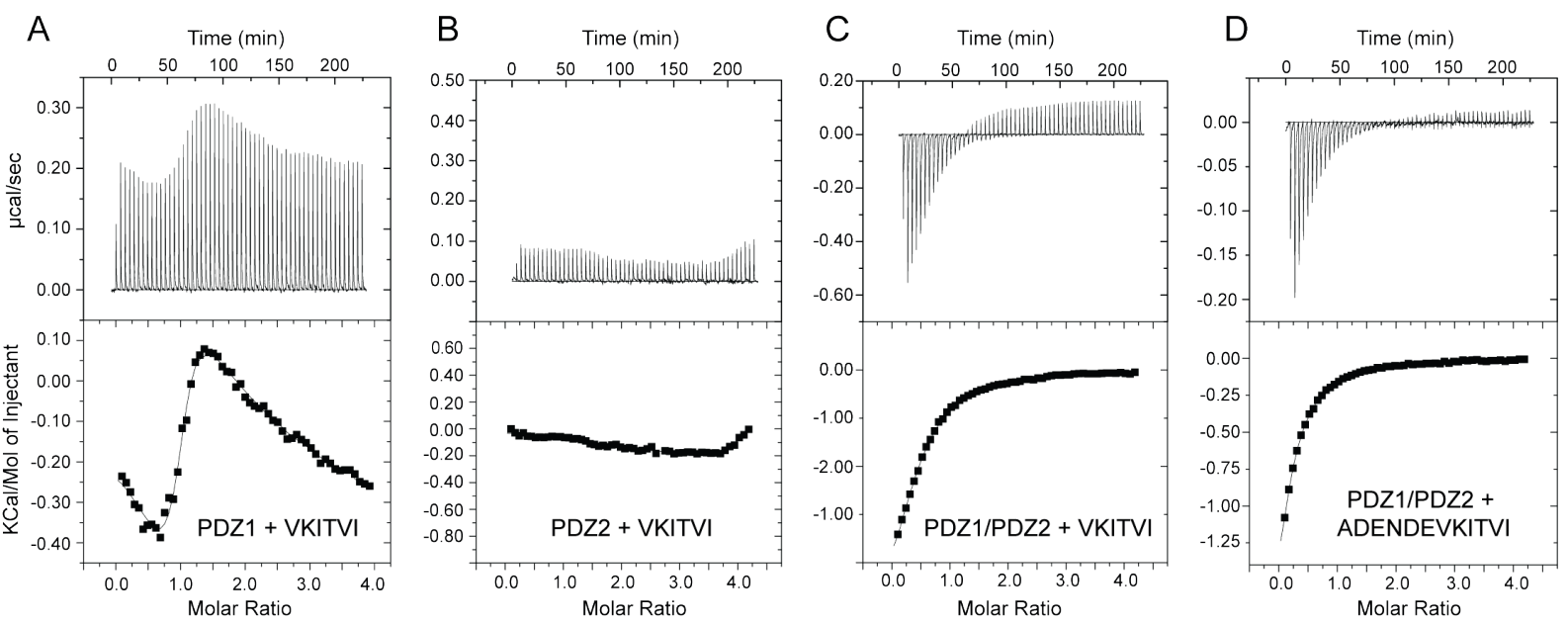

Figure 4. Titration of GRASP65 constructs with the GM130 peptides NVKITVIC and NADENDEVKITVIC. (A) GRASP65 ${ }^{\text {PDZ1 }}$ titration with ${ }^{N}$ VKITVIC peptide. The data were fitted to a sequential binding site model with 4 binding sites and revealed a $K_{D}=41.8 \pm$ $2.97 \mu \mathrm{M}, \Delta H=-369.9 \pm 12.0 \mathrm{cal} / \mathrm{mol}, \Delta S=18.7 \mathrm{cal} / \mathrm{mol} / \mathrm{deg}$. (B) The GRASP65 PDZ2 titration with NVKITVIC GM130 peptide did not show any binding. (C, D) The titration of the GRASP domain (GRASP651-204) is shown with GM130 NVKITVIC peptide in C) and ${ }^{N} A D E N D E V K I T V I C$ in D). The following thermodynamic parameters were determined for (C) $K_{D}=25.06 \pm 0.63 \mu \mathrm{M}, \Delta H=-6427 \pm$ $120.3 \mathrm{cal} / \mathrm{mol}, \Delta S=-1.25 \mathrm{cal} / \mathrm{mol} / \mathrm{deg}$ and $N=0.46$ sites; for $(D) K_{D}=27.62 \pm 0.59 \mu \mathrm{M}, \Delta H=-4160 \pm 212.4 \mathrm{cal} / \mathrm{mol}, \Delta S=6.43$ $\mathrm{cal} / \mathrm{mol} / \mathrm{deg}$ and $N=0.17$ sites. 
A

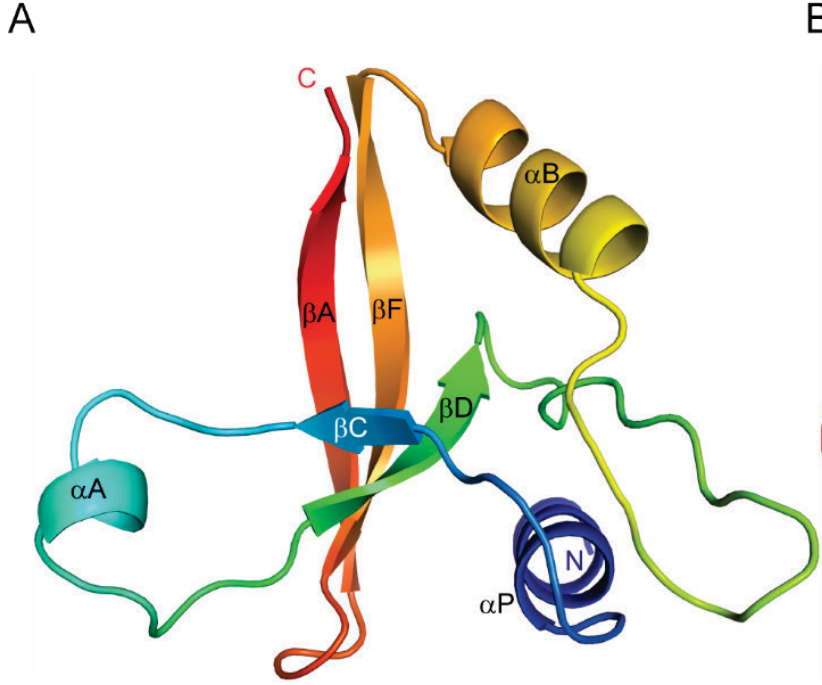

B

C

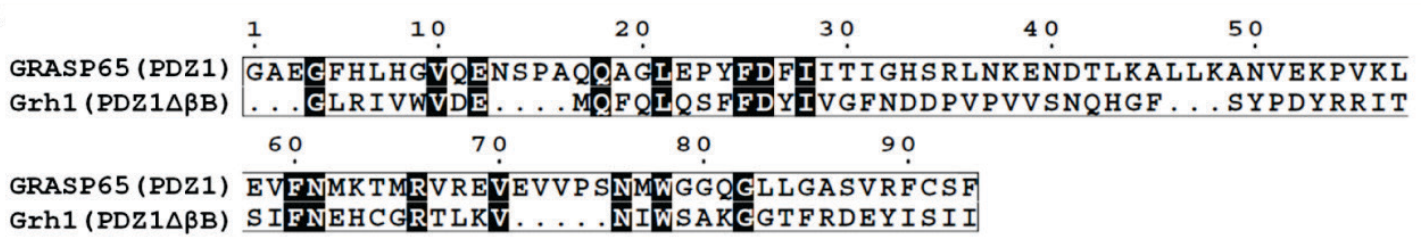

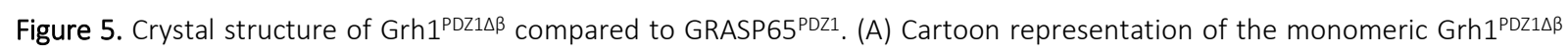
structure, color-coded from the $\mathrm{N}$-terminus in blue to the $\mathrm{C}$-terminus in red. Helix $\alpha \mathrm{P}$ belongs to the Prescission protease recognition site. The construct consists of four $\beta$-strands ( $\beta A, \beta C, \beta D$ and $\beta F)$, three $\alpha$-helices $(\alpha A, \alpha B$ and $\alpha P)$ and an extended loop region connecting strand $\beta D$ with helix $\alpha B$. The uncleaved $\mathrm{His}_{6}$ tag was not visible in the electron density. (B) Superposition

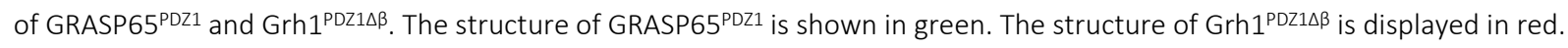
The black arrow shows the putative position of the missing strand $\beta B$ of Grh1 $1^{\text {PDZ1 } \triangle \beta}$ guided by the position of $\beta B$ from GRASP65 ${ }^{\mathrm{PDZ1}}$. The dashed line indicates the approximate position of the missing carboxylate-binding loop and the C-terminus completing the PDZ of Grh1. For reasons of clarity the N-terminal helices $\alpha$ L from GRASP65 PDZ1 and $\alpha$ P from Grh $1{ }^{67-114}$ are not shown. (C) Sequence alignment of both PDZ domains comprising residues 13-105 for GRASP65 PDZ1 and 69-144 for Grh1PDZ1AB as shown in the structure in (B).

of GRASP65, but in slightly incomplete form lacking the last $\beta$ strand. Despite very low sequence identity of only $10 \%$, Grh1 $1^{\text {PDZ1 } \triangle \beta B}$ also shows a PDZ folding pattern indicating structural conservation that is not reflected in the amino acid sequence. Circular permutations of protein sequences are frequently observed and may occur in different protein folds. ${ }^{[39,40]}$

To characterize the binding of GM130 to GRASP65 in detail, we used the isothermal titration calorimetry method. Instead of in vitro translated protein constructs or GST-tagged GM130 fragments, ${ }^{[8,13]}$ we used untagged GM130 peptides for the ITC measurements and combined them with various recombinantly expressed GRASP65 constructs using either the single PDZ domains or the entire GRASP domain. In contrast to earlier observations ${ }^{[13]}$ we could not verify that GRASP65 ${ }^{\text {PDZ2 }}$ alone is sufficient for
GM130 binding. The same observation was recently made by Hu et al. who showed that only the GRASP65 PDZ1 and PDZ2 in tandem are capable of GM130 binding, where the first PDZ domain is the key player in this molecular interaction. ${ }^{[12]}$ Our own ITC experiment reveals a peptide length of six amino acids (VKITVI) of the GM130 C-terminus for sufficient binding to the isolated first PDZ domain and to the entire GRASP domain GRASP1-204, but not to the isolated second PDZ domain of GRASP65. The GM130 dodecapeptide ADENDEVKITVI bound to the entire GRASP domain with a $K_{D}$ of $27.6 \mu \mathrm{M}$, i.e. with a similar affinity as observed with the GM130 hexapeptide $\left(K_{D}=25.2 \mu \mathrm{M}\right)$. That indicates that the additional amino acids more distant from the $\mathrm{C}$ terminus of GM130 do not significantly contribute in binding to the GRASP domain. These additional residues form an exposed loop and are not involved in binding to 
GRASP65 as observed in the crystal structure of Hu et al.[12] There, a 22-residue peptide NSNPCIPFFYRADENDEVKITVIC is bound to the GRASP domain with a $K_{D}$ of $108 \mathrm{nM}$. This 230 times lower $K_{D}$ as compared to the hexa- and dodecapeptides examined in the current study is likely to result from additional hydrophobic residues present in the longer peptide, which are binding into the strongly hydrophobic inter-domain pocket of the GRASP domain. Our measured $\mathrm{K}_{\mathrm{D}}$ reflects the contribution of the conventional PDZ domain recognition mode to the overall GM130-GRASP65 interaction.

Author Contributions. C.M.J. designed the biochemical studies and performed cloning, purification and crystallization experiments. C.M.J. and Y.R. collected X-ray diffraction data, solved and refined the structures. C.M.J. and Y.R. wrote the manuscript. U.H. contributed to revising intellectual content, finalized the manuscript, and holds responsibility for the integrity of the data as corresponding author. All authors approved the final manuscript.

Competing Interests. The authors declare no competing interests.

Acknowledgment. We wish to thank Drs. Harald Striegl, Katja Fälber and Marcel Jurk for scientific discussions and helpful advice and Dr. Ulrich Gohlke for critical reading of the manuscript. Furthermore, we thank Professors Erich Wanker and Thomas Sommer (both from the Max Delbrück Center for Molecular Medicine, Berlin) for kindly providing us with the cDNA for GRASP65 and Grh1. Diffraction data were collected on BL14.1 at the BESSY II electron storage ring operated by the Helmholtz-Zentrum Berlin, Germany. We particularly acknowledge the help and support of Drs. Uwe Mueller and Manfred Weiss during the experiment. This work was funded by the Sonderforschungsbereich 740 (SFB740) of the German Research Foundation (DFG).

\section{REFERENCES}

[1] F. A. Barr, B. Short, Curr. Opin. Cell Biol. 2003, 15, 405.

[2] I. B. Ramirez, M. Lowe, Semin. Cell Dev. Biol. 2009, 20, 770 .

[3] C. Rabouille, A.D. Linstedt, Front Cell Dev. Biol. 2016, $4,1$.

[4] J. Shorter, R. Watson, M. E. Giannakou, M. Clarke, G. Warren, F. A. Barr, EMBO J. 1999, 18, 4949.

[5] F. Barr, M. Puype, J. Vandekerckhove, G. Warren, Cell 1997, 91, 253.

[6] B. Short, C. Preisinger, R. Körner, R. Kopajtich, O. Byron, F. A. Barr, J. Cell Biol. 2001, 155, 877.

[7] M. A. Puthenveedu, C. Bachert, S. Puri, F. Lanni, A. D.
Linstedt, Nat. Cell Biol. 2006, 8, 238.

[8] C. Bachert, A. D. Linstedt, J. Biol. Chem. 2010, 285, 16294.

[9] Y. Wang, A. Satoh, G. Warren, J. Biol. Chem. 2005, 280, 4921.

[10] Y. Xiang, Y. Wang, J. Cell Biol. 2010, 188, 237.

[11] T. N. Feinstein, A. D. Linstedt, Mol. Biol. Cell 2008, 19, 2696.

[12] F. Hu, X. L. Shi, B. W. Li, X. C. Huang, X. Morelli, N. Shi, J. Biol. Chem. 2015, 290, 26373.

[13] F. Barr, N. Nakamura, G. Warren, EMBO J. 1998, 17, 3258.

[14] F. Jeleń, A. Oleksy, K. Smietana, J. Otlewski, Acta Biochim. Pol. 2003, 50, 985.

[15] B. J. Hillier, K. S. Christopherson, K. E. Prehoda, D. S. Bredt, W. A. Lim, Science 1999, 284, 812.

[16] J. Saupe, Y. Roske, C. Schillinger, N. Kamdem, S. Radetzki, A. Diehl, H. Oschkinat, G. Krause, U. Heinemann, J. Rademann, ChemMedChem. 2011, 6, 1411.

[17] H. J. Lee, J. J. Zheng, Cell Commun. Signal. 2010, 8, 8 .

[18] C. Aslanidis, P. J. de Jong, Nucleic Acids Res. 1990, 18, 6069.

[19] U. Heinemann, K. Bussow, U. Mueller, P. Umbach, Acc. Chem. Res. 2003, 36, 157.

[20] W. Kabsch, Acta Crystallogr. D Biol. Crystallogr. 2010, 66, 125.

[21] M. Krug, M.S. Weiss, U. Heinemann, U. Mueller, J. Appl. Crystallogr. 2012, 45, 568.

[22] A. J. McCoy, R. W. Grosse-Kunstleve, P. D. Adams, M. D. Winn, L. C. Storoni, R. J. Read, J. Appl. Crystallogr. 2007, 40, 658.

[23] M. D. Winn, C. C. Ballard, K. D. Cowtan, E. J. Dodson, P. Emsley, P. R. Evans, R. M. Keegan, E. B. Krissinel, A. G. Leslie, A. McCoy, S. J. McNicholas, G. N. Murshudov, N. S. Pannu, E. A. Potterton, H. R. Powell, R. J. Read, A. Vagin, K. S. Wilson, Acta Crystallogr. D Biol. Crystallogr. 2011, 67, 235.

[24] Collaborative Computational Project, Number 4, Acta Crystallogr. D Biol. Crystallogr. 1994, 50, 760.

[25] P. Emsley, B. Lohkamp, W.G. Scott, K. Cowtan, Acta Crystallogr. D Biol. Crystallogr. 2010, 66, 486.

[26] P. Emsley, K. Cowtan, Acta Crystallogr. D Biol. Crystallogr. 2004, 60, 2126.

[27] G. N. Murshudov, A. A. Vagin, E. J. Dodson, Acta Crystallogr. D Biol. Crystallogr. 1997, 53, 240.

[28] G. N. Murshudov, P. Skubák, A. A. Lebedev, N. S. Pannu, R. A. Steiner, R. A. Nicholls, M. D. Winn, F. Long, A. A. Vagin, Acta Crystallogr. D Biol. Crystallogr. 2011, 67, 355.

[29] B. C. Wang, Methods Enzymol. 1985, 115, 90.

[30] W. A. Hendrickson, M. M. Teeter, Nature 1981, 290, 107. 
[31] S. Panjikar, V. Parthasarathy, V. S. Lamzin, M. S. Weiss, P. A. Tucker, Acta Crystallogr. D Biol. Crystallogr. 2005, 61, 449.

[32] A. Perrakis, R. Morris, V. S. Lamzin, Nat. Struct. Biol. 1999, 6, 458.

[33] V. B. Chen, W. B. Arendall, J. J. Headd, D. A. Keedy, R. M. Immormino, G. J. Kapral, L. W. Murray, J. S. Richardson, D. C. Richardson, Acta Crystallogr. D Biol. Crystallogr. 2010, 66, 12.

[34] S. Keller, C. Vargas, H. Zhao, G. Piszczek, C. A. Brautigam, P. Schuck, Anal. Chem. 2012, 84, 5066.

[35] E. Krissinel, K. Henrick, J. Mol. Biol. 2007, 372, 774.

[36] R. Behnia, F. A. Barr, J. J. Flanagan, C. Barlowe, S. Munro, J. Cell Biol. 2007, 176, 255.
[37] S. K. Levi, D. Bhattacharyya, R. L. Strack, J. R. Austin, B. S. Glick, Traffic 2010, 11, 1168.

[38] S. T. Truschel, D. Sengupta, A. Foote, A. Heroux, M. R. Macbeth, A. D. Linstedt, J. Biol. Chem. 2011, 286, 20125.

[39] U. Heinemann, M. Hahn, Prog. Biophys. Mol. Biol. 1995, 64, 121.

[40] U. Heinemann, M. Hahn, Trends Biochem. Sci. 1995, 20, 349 .

[41] N. A. Baker, D. Sept, S. Joseph, M. J. Holst, J. A. McCammon, Proc. Natl. Acad. Sci. USA 2001, 98, 10037.

[42] P. Gouet, E. Courcelle, D. I. Stuart, F. Métoz, Bioinformatics 1999, 15, 305. 